\title{
An efficient framework for the automatic and dynamic load distribution in IOT
}

\author{
Vijaita Kashyap* and Dimple Kapoor \\ Assistant Professor, Department of Computer Science and Engineering, Chitkara University, Rajpura, Punjab
}

Received: 20-October-2019; Revised: 26-December-2019; Accepted: 28-December-2019

C2019 Vijaita Kashyap and Dimple Kapoor. This is an open access article distributed under the Creative Commons Attribution (CC BY) License, which permits unrestricted use, distribution, and reproduction in any medium, provided the original work is properly cited.

\begin{abstract}
The current era is observing the need of communication among different smart devices in the collaboration of Internet of Things (IoT). Smart device integration along with the load distribution is capable in controlling energy resources with the cost benefits. So, in this paper an efficient framework for the automatic and dynamic load distribution in IOT with smart grids mechanism has been presented. Our efficient dynamic load balancing framework has three phases. First shows the pre-processing, second phase shows the IoT distribution and communication procedure. Final phase is the object interconnection phase with grids. For the evaluation of our framework scaling mechanism has been adopted for testing of load clusters. The results indicate that it is capable in energy resource saving as it found to be uniform.
\end{abstract}

\section{Keywords}

IoT, Data mining, Big data, Cloud computing, Computation capability.

\section{Introduction}

In today's world data sharing and communication is very important, especially with the smart devices and grids. Internet of Things (IoT) provides a communication network [1]. The device connection and interconnection depend on the object availability and the connection enhancements applicability [2]. It also provides interconnection between different devices for data sharing through the web environment. The role of IoT is mainly important in the area where the data and resource distribution and communication between sensor, smart devices and smart grids [3-5]. The coverage area is also very wide in case of IoT devices which includes healthcare industry, visualization area, smart monitoring system etc. [5]. The devices used in the IoT are generally supports the heterogeneity with other smart devices. So, data sensitivity is also a crucial aspect in these devices. Data sensitivity can be handled in the way suggested in [6]. For the message transmission and control, there are several protocols which can be used and different objects in terms of communication devices have been used. The research applicability can be extended with data mining, Big Data and cloud computing techniques, etc. $[7,8]$.

*Author for correspondence

267
If we think about the smart grid mechanism in IoT then in general it is a part of the complete IoT framework. It has been used for the remote monitoring and management [9]. It is helpful in different areas including the congestion control in traffic and for warning systems [9]. In [10] authors have suggested the smart grids have been in demand due to the traditional grid's drawbacks like energy demand, wastage and security issues. These are the factors which arise the need of smart grid framework. The major aspects covered in the case of smart grids are the network for the data communication [11]. It provides the integration of the data analysis, covering and acquiring the transmission lines and distribution substations [11]. It also provides the full integration of the power grid. The main work is the data collection and analysis [11]. The components used are in the area suggested and discussed above covers the need of the current scenario also. It also shows that the devices have been increased with the connected devices in terms of positive relationship [12]. It also reveals the increasing impact of smart devices and grids in the IoT framework [13].

The main objectives concentrating the limitations are as follows: 
1. To study and analyses the dynamic load distribution scenario in the current IOT based smart grid system.

2. To propose an efficient framework for automatic and dynamic load distribution in IOT based smart grids.

3. To evaluate the applicability of the above framework and their implementation aspects. These aspects will be helpful in the creation of the future framework and development.

\section{Related work}

In 2018, Barros et al. [14] have been used fog computing for the logical distance reduction. It has been calculated between the central distribution and consumption spot. It is more effective. They have also focused on the power flow information. They have evaluated the performance of the NewtonRaphson and Gauss-Seidel algorithms. The main objective is to develop calculations. It has been developed for the load flow problem. It has been done with the help of fog. Their results show the possibility of making the smart grid based on fog computing, which is capable of reacting the environment.

In 2018, Fan and Ansari [15] discussed about the edge computing. It has the capability of data's ability based on IoT devices. They have proposed traffic load balancing scheme. It is capable in minimizing the wireless latency in case of IoT users. They have divided the problem into two sub problems. Based on that they have designed two algorithms. Their results support their approach.

In 2018, Wang et al. [16] discussed about machinetype communication (MTC). It has been discussed in terms of $5 \mathrm{G}$ network. They have proposed an energyefficient clustering routing algorithm. They have proposed uneven cluster formation scheme. It has been done for the load balancing and energy efficiency. It has been considered for the nonuniform traffic distribution. They have proposed a distributed cluster head $(\mathrm{CH})$ rotation mechanism. It has the capability of energy consumption balancing within each cluster. Their result shows that it is efficient in terms of network lifetime, throughput and energy efficiency.

In 2019, Hribar and DaSilva [17] have been proposed a mechanism based on the updating and learning. The collected content of information has the capability of learning. Their approach is capable in the frequency reduction. Correlated information has been used along with the evaluation mechanism. The efficiency proved to be increasing through their approach.

In 2019, Mostafa [18] presented and analyzed the mechanism for the quality control arrangements with the insights of IoT security and system performance mechanism. They have also shown the detail analysis on them for the future tracks.

In 2019, Muhammad et al. [19] discussed about machine to machine communication mechanism in terms of IoT system. They have also focused on security mechanism. They have presented the implementation, development framework of secure message queuing telemetry transport (MQTT). They have also suggested and discussed the security for the different IoT applications. For testing the approach, they have examined and considered the smart home real world application.

In 2019, Munsadwala et al. [20] discussed the air pollution in terms of tracking and detection approach. They have also discussed the exponential growth and causes. They have discussed in terms of sensor with a GPS system in terms of IoT. They have used MQTT protocol, which has been helpful in the data acquisition unit. They have also provided the dashboard for the stored data visualization. They have also provided the email notifications, activity $\log$, geographic location verification and device authentication functionalities. They have suggested that based on this the authorized user has the capability of control from the remote location.

In 2019, Poulter et al. [21] have been presented their view on the secure remote update protocol. It has been applied to the secure message's transmission formation. They have discussed the creation of Python wrapper for SRUP. According to the authors this feature has the capability of the need of writing less code. It also shows minimal efforts requirement.

In 2019, Alhazmi and Aloufi [22] discussed and explored the IoT architecture, applications and needs. They have suggested the adoption of traditional data center or cloud computing based data center for the IoT. They have suggested that the main limitation through the cloud computing based data center is it is very far. So, they have suggested fog based IoT. They have suggested that it can be helpful in faster analysis, so it is better for the time-sensitive applications. They have suggested that it will be helpful in the adoptability of in different security aspects. It can be based on the resources available 
and adoptability with the different performance constraints. They have discussed the respective challenges along with the performance analysis. Their proposed security scheme relies on MQTT protocol. They have discussed in terms of sensor with a GPS system in terms of IoT. They have used MQTT protocol which has been helpful in the data acquisition unit.

A lot of research has been conducted in various applications of IoT, but electric grids are comparatively underexplored. As studied in the literature survey, most of the research conducted in this area is limited to the implementation of smart electricity meters in smart homes. The conventional electricity grids and smart grids under the lens simply rely on manual management of electricity supply. Not much work has been done with the focus on the dynamic load distribution of electricity. The literature survey conducted suggests that there is a scope in developing a dynamic load distribution system of electricity which can automatically manage the electric supply. Therefore, in this research a novel approach will be developed for automatic and dynamic load distribution in smart grids.
Our efficient dynamic load balancing framework has been divided in three phases. Figure 1 shows the IoT pre-processing mechanism. In this complete communication along with the access mechanism has been applied. It includes aggregation, load sharing and computation. It includes a data access mechanism with the similar pairs of data activity for the data sharing. It provides the data aggregation, pre-processing and computation process. Figure 2 shows the IoT distribution and communication procedure. It shows the process of connection, segmentation and load balancing. Figure 3 shows the IoT connection procedure in the final phase.

In the first phase data mining approaches like clustering and association rule mining can be helpful for the distribution and arrangement. In the second phase object detection mechanism with classification approaches have been applied and validated. In the third or the final phase optimization along with the misclassification result handling approaches may be useful and applicable for the finding of the calculative and computational efficiency. It will be extended with the attribute property validation and object connection removal.

\section{Methods and framework}

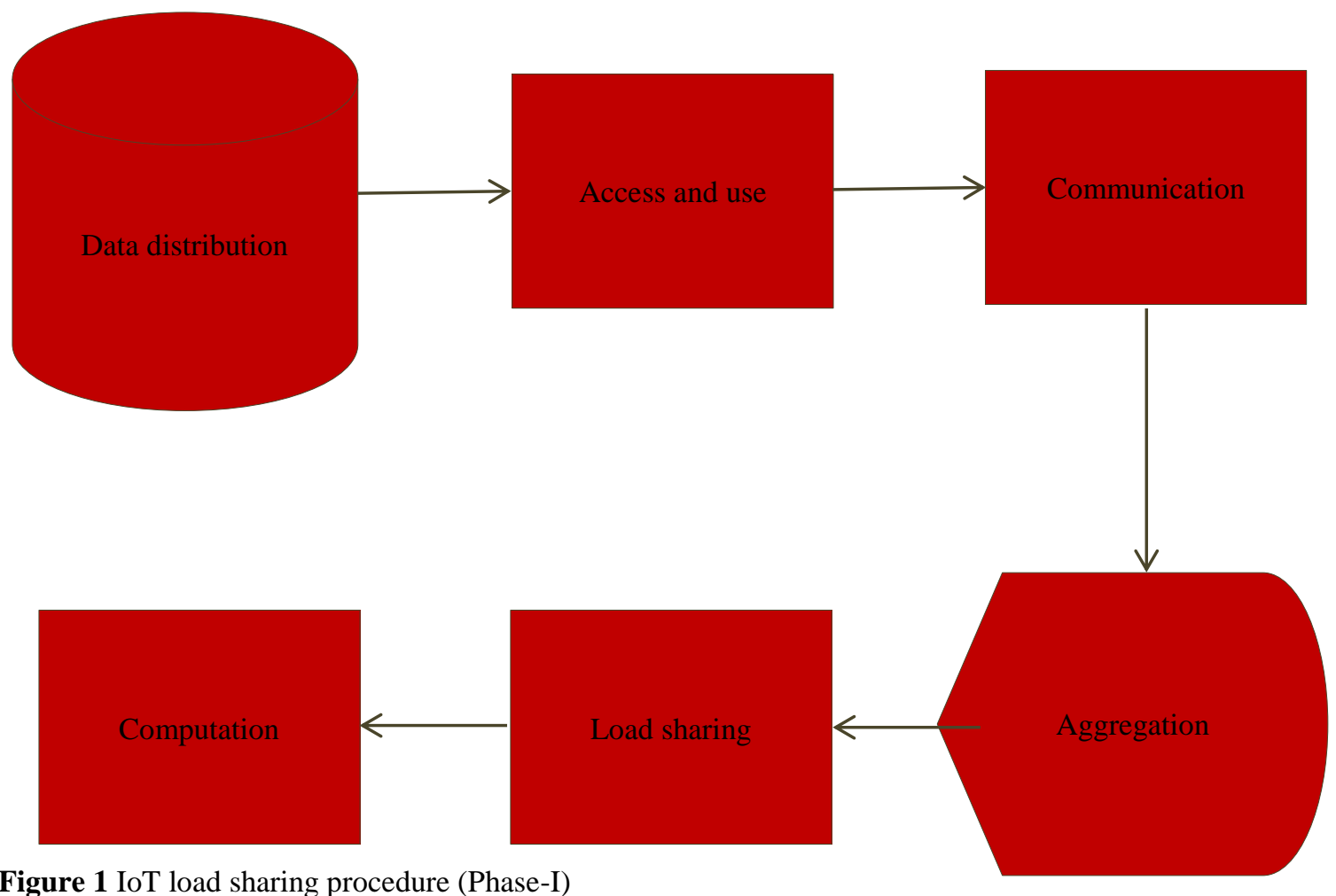



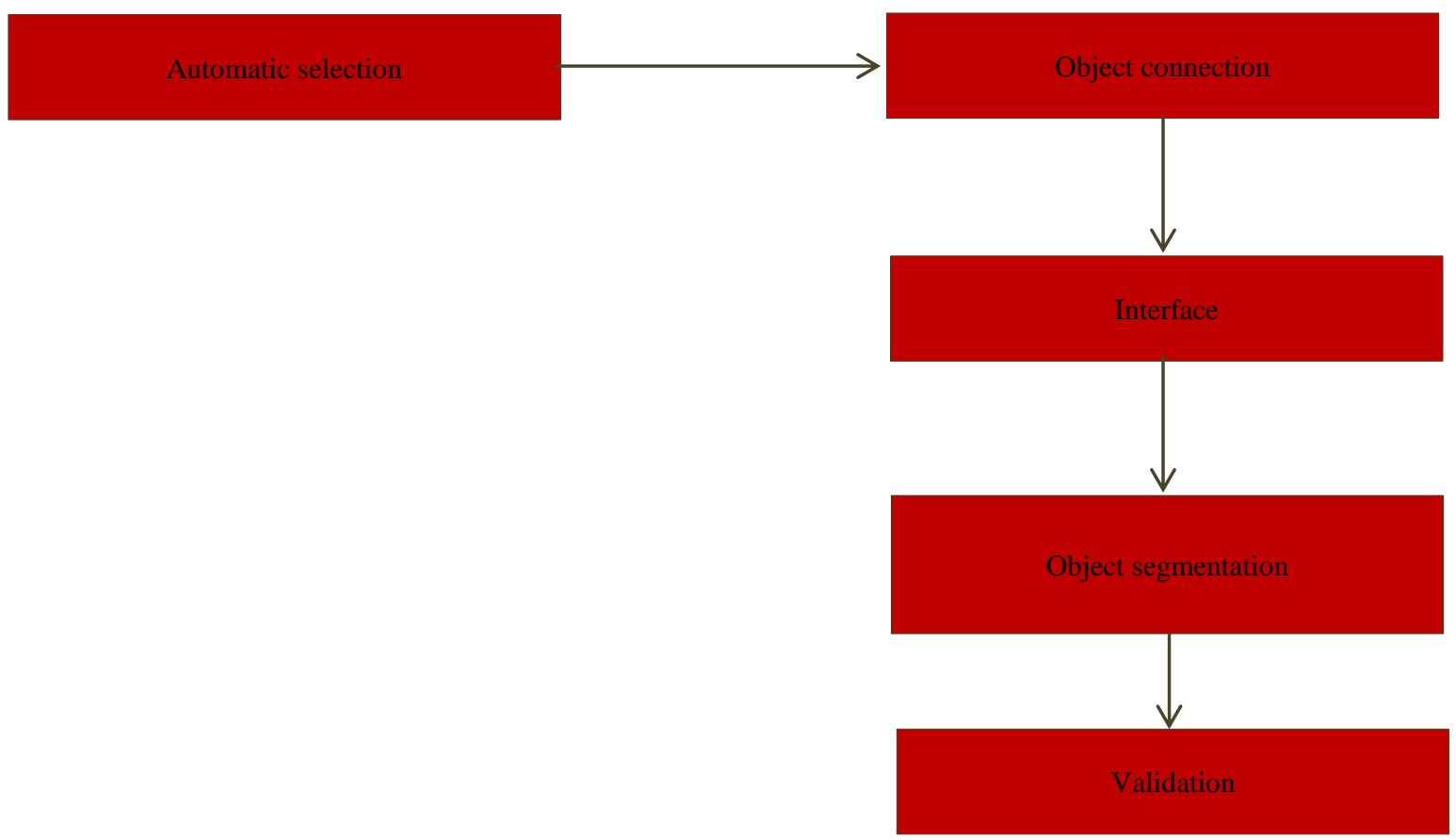

Figure 2 IoT distribution and communication procedure (Phase-II)

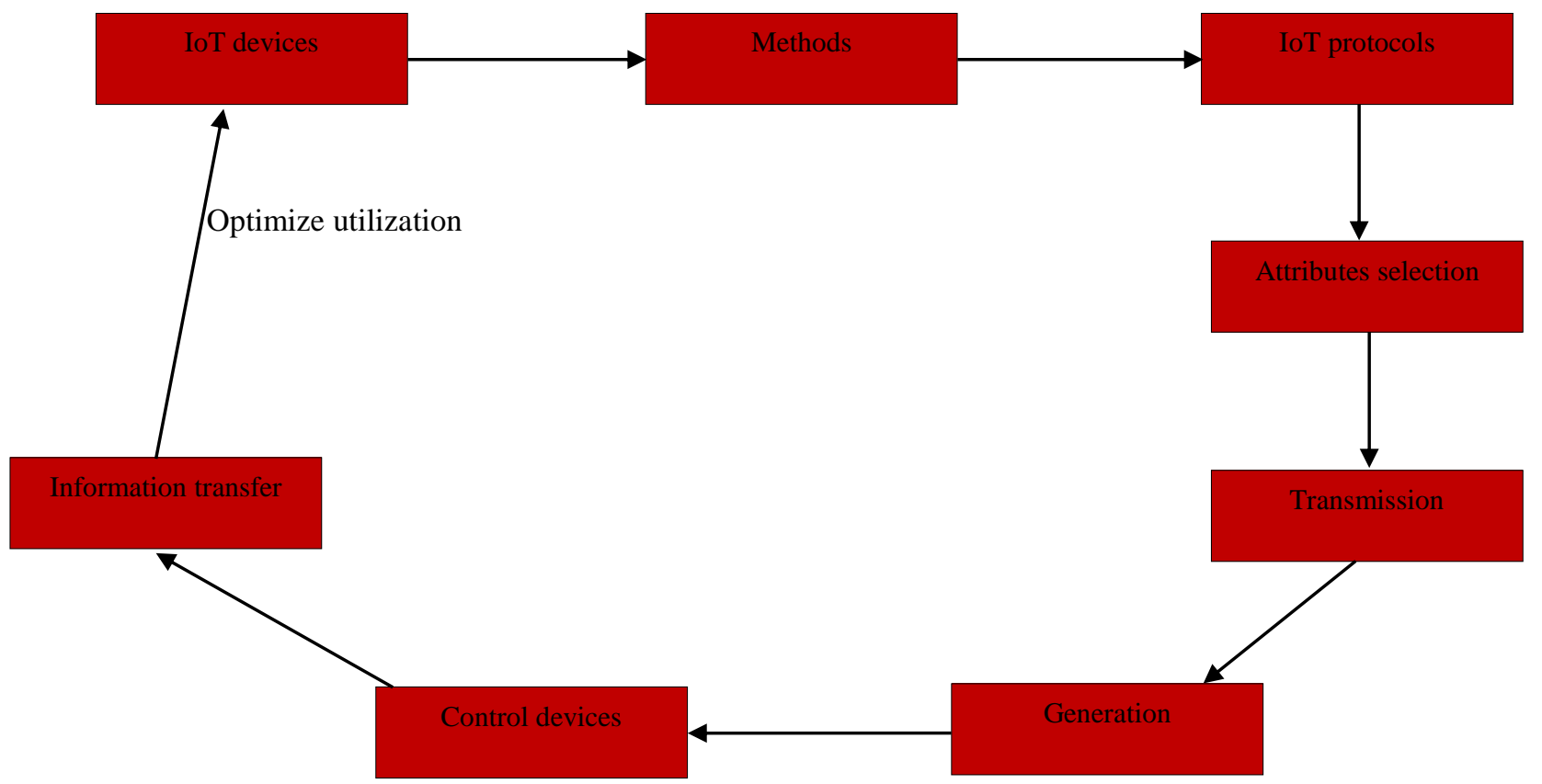

Figure 3 IoT connection procedures (Phase-III)

The following steps can be the part of our proposed framework and the study.

Step 1: In this an extensive study of the dynamic load distribution approach of energy in smart grid will be conducted and it will lead to the improvements in the smart grid systems. Various existing approaches that can be used on IOT in the field of smart grid management, which can improve efficiency will be studied thoroughly.

Step 2: As the Smart grid comprises of different hardware's at end nodes, therefore there might be some compatibility issues. So, in this phase thorough 
study of different hardware is done and the optimum one according to our requirements is chosen. The Hardware includes different sensors, plugs, end devices etc.

Step 3: A novel approach is developed which can learn from historical and cloud database and then manage the smart grid effectively and efficiently.

Step 4: The approach developed will be Deployed/ Simulated. It can be done either using available hardware or on different simulators.

Step 5: The results of the proposed work will be analyzed on different parameters like Energy Conserved, Cost, Loss in transmission, Peak to Average Ratio (PAR), System Robustness etc.

Step 6: The performance of the novel approach shall be compared with the existing approaches. The comparison will provide the pros and cons of the proposed approach.

\section{Results and discussion}

Smart grids are the integral part of smart cities. The prevalent methods of load distribution are static in nature and lead to wastage of resources. The proposed work aims at providing a solution to this by implementing automatic and dynamic load distribution, which in turn will result in effective use of resources by reducing the energy wastage and cost cutting. Implementation of the proposed approach will result in a more robust and efficient electricity distribution system, which in turn leads to reduction of carbon footprint. For the initial testing, scaling has been performed. First, scaling has been performed for the considered cluster 1 load balancing and the second is considered for cluster 2 load balancing. Figure 4 shows the load balancing for the one cluster. Figure 5 shows the load balancing for the two clusters. It clearly shows the uniformity.

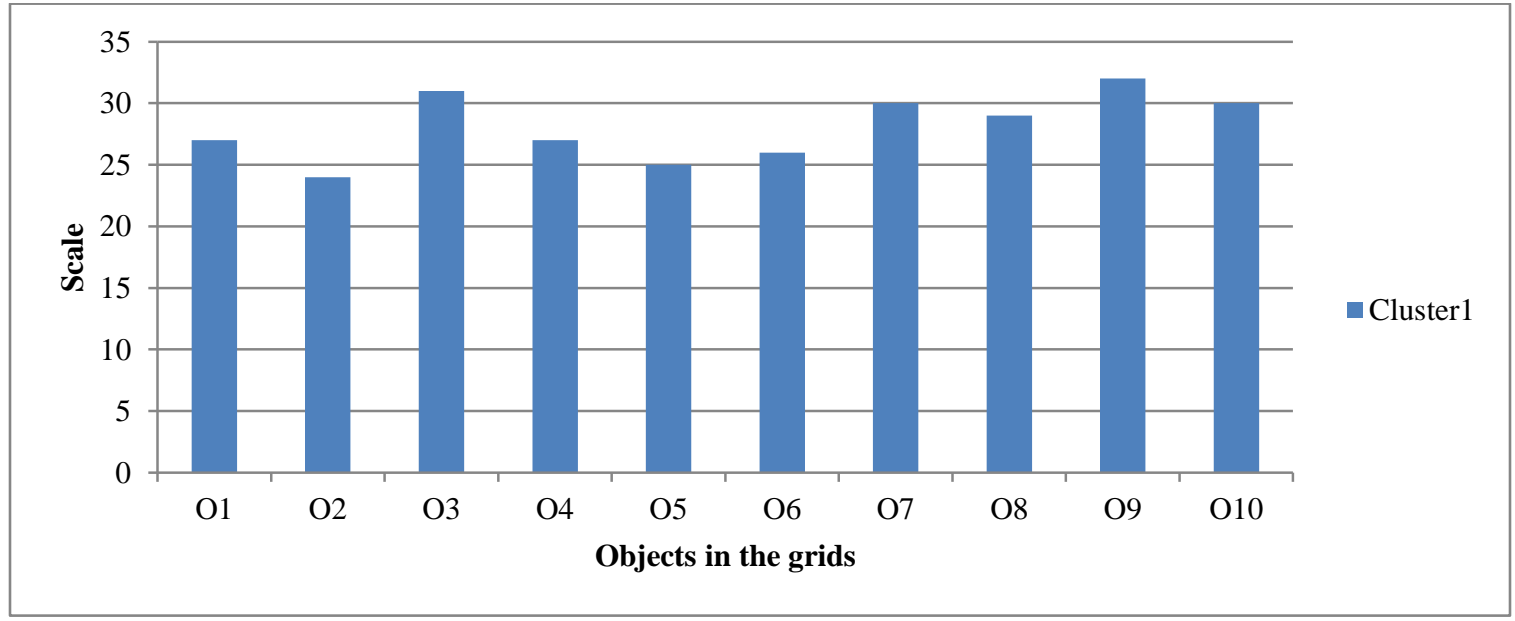

Figure 4 Scaling for the load cluster 1

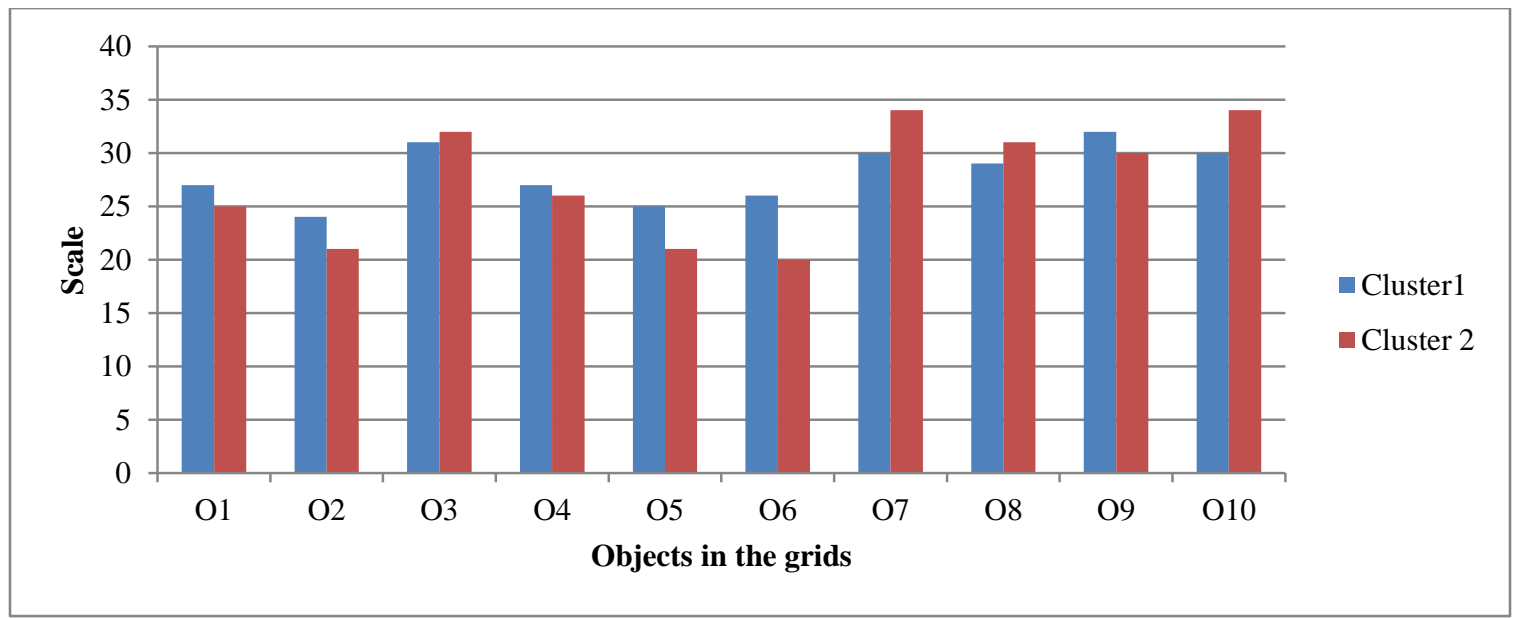

Figure 5 Scaling for the load cluster 2 


\section{Conclusions}

This paper is divided mainly into three parts. First part explores the objectives along with the study, which focuses on the gaps identified. Second part explored the framework with their phases and the implementation strategy. The capable methods have been discussed for each phase with the strategy for the consideration of the approaches and their applicability. In the final phase the framework applicability has been tested with the scale and their adoptability is considered for the future work.

\section{Acknowledgment}

None.

\section{Conflicts of interest}

The authors have no conflicts of interest to declare.

\section{References}

[1] Tyson G, Sastry N, Rimac I, Cuevas R, Mauthe A. A survey of mobility in information-centric networks: challenges and research directions. In proceedings of the ACM workshop on emerging name-oriented mobile networking design-architecture, algorithms, and applications 2012 (pp. 1-6). ACM.

[2] Johnson S, Fayaz MA, Krishnan HS. IoT based rearend collision avoidance system in highways. International Journal of Advanced Computer Research. 2019; 9(45):379-85.

[3] Arshad S, Azam MA, Rehmani MH, Loo J. Recent advances in information-centric networking-based internet of things (ICN-IoT). IEEE Internet of Things Journal. 2018; 6(2):2128-58.

[4] Jin Y, Gormus S, Kulkarni P, Sooriyabandara M. Content centric routing in IoT networks and its integration in RPL. Computer Communications. 2016; 89:87-104.

[5] Patel P, Patel HB, Shrimali B. Data delivery techniques in content centric routing for IoT: a systematic review. International Journal of Advanced Technology and Engineering Exploration. 2018; 5(48):445-51.

[6] Dubey AK. An efficient fuzzy C-means method with variable FV-TC for Data sensitivity calculation in a cloud computing environment. International Journal of Engineering and Advanced Technology.2019; 9(1):4486-90.

[7] Mishra A, Mohapatro M. An IoT framework for biomedical sensor data acquisition and machine learning for early detection. International Journal of Advanced Technology and Engineering Exploration.2019; 6(54):112-25

[8] Dubey AK, Dubey AK, Agarwal V, Khandagre Y. Knowledge discovery with a subset-superset approach for mining heterogeneous data with dynamic support. In CSI sixth international conference on software engineering 2012 (pp. 1-6). IEEE.
[9] https://www.telit.com/blog/iot-smart-grid-benefits/ . Accessed 12 July 2019.

[10] Saleem Y, Crespi N, Rehmani MH, Copeland R. Internet of things-aided smart grid: technologies, architectures, applications, prototypes, and future research directions. IEEE Access. 2019; 7:629623003.

[11] Ghasempour A. Internet of things in smart grid: architecture, applications, services, key technologies, and challenges. Inventions. 2019; 4(1):1-12.

[12] Ahmed HI, Nasr AA, Abdel-Mageid S, Aslan HK. A survey of IoT security threats and defenses. International Journal of Advanced Computer Research. 2019; 9(45):325-50.

[13] Tsai CW, Lai CF, Vasilakos AV. Future internet of things: open issues and challenges. Wireless Networks. 2014; 20(8):2201-17.

[14] Barros E, Peixoto M, Leite D, Batista B, Kuehne B. A fog model for dynamic load flow analysis in smart grids. In symposium on computers and communications 2018 (pp. 1-6). IEEE.

[15] Fan Q, Ansari N. Towards traffic load balancing in drone-assisted communications for IoT. IEEE Internet of Things Journal. 2018; 6(2):3633-40.

[16] Wang Z, Qin X, Liu B. An energy-efficient clustering routing algorithm for WSN-assisted IoT. In wireless communications and networking conference 2018 (pp. 1-6). IEEE.

[17] Hribar J, DaSilva L. Utilising correlated information to improve the sustainability of internet of things devices. In world forum on internet of things (WFIoT) 2019 (pp. 805-8). IEEE.

[18] Mostafa B. Monitoring internet of things networks. In world forum on internet of things 2019 (pp. 295-8). IEEE.

[19] Muhammad A, Afzal B, Imran B, Tanwir A, Akbar AH, Shah G. OneM2M architecture based secure MQTT binding in Mbed OS. In European symposium on security and privacy workshops 2019 (pp. 48-56). IEEE.

[20] Munsadwala Y, Joshi P, Patel P, Rana K. Identification and visualization of hazardous gases using IoT. In international conference on internet of things: smart innovation and usages 2019 (pp. 1-6). IEEE.

[21] Poulter AJ, Johnston SJ, Cox SJ. PySRUPsimplifying secure communications for command \& control in the internet of things. In world forum on internet of things 2019 (pp. 273-7). IEEE.

[22] Alhazmi OH, Aloufi KS. Fog-based internet of things: a security scheme. In international conference on computer applications \& information security. 2019 (pp. 1-6). IEEE. 
International Journal of Advanced Technology and Engineering Exploration, Vol 6(61)

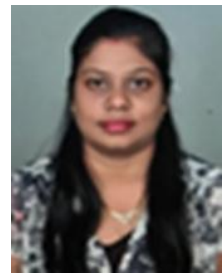

Vijaita Kashyap received her Master in Technology degrees in Computer Science and Engineering from Samrat Ashok Technological University, Vidisha, M.P in 2012. She has currently served as an Assistant Professor in the Department of Computer Science and Engineering at Chitkara University, Rajpura, Punjab. Her research interests include Data Mining and IOT.

Email: vijaita.kashyap@chitkara.edu.in

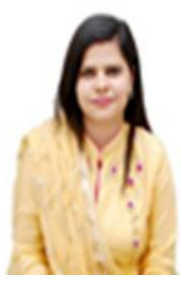

Dimple Kapoor received her Master in Technology degrees in Computer Science and Engineering from Meerut Institute of Engineering \& Technology, Meerut, U.P in 2015. She has currently served as an Assistant Professor in the Department of Computer Science and Engineering at Chitkara University, Rajpura, Punjab. Her research interests include Image Processing, Data Mining and IOT. 